\title{
La transnacionalización de la educación superior: ¿qué papel juegan los nuevos regionalismos en la difusión de estas ideas? El caso del MERCOSUR (1992-2012) en perspectica comparada
}

\author{
Mercedes Botto
}

\section{RESUMEN}

El objetivo de este trabajo es contribuir a la comprensión de la reforma de la educación superior de los años noventa en América Latina, analizando la influencia ejercida por los procesos de integración regional, más precisamente el MERCOSUR. ¿En qué medida ayudó a difundir y poner en marcha las ideas de transnacionalización o de internacionalización que estaban en debate a nivel global? Para responder a esta pregunta, usaremos la comparación de la experiencia del MERCOSUR. con la de otros regionalismos, identificando las iniciativas en torno a la movilidad de estudiantes y a la acreditación de carreras, dos iniciativas que abordan por igual todos los procesos de integración analizados. La conclusión a la que llegamos sugiere que los nuevos regionalismos avanzaron a una tercera posición: defenderse de la globalización a través de políticas de cooperación solidaria, al tiempo que buscaban conformar un espacio regional de educación superior desde donde competir globalmente. En América del Sur, sin embargo, los avances se han reducido hasta el momento a consolidar las capacidades nacionales, promoviendo los procesos de reforma inconclusos en los países menos desarrollados y consolidando las posiciones estratégicas y dominantes de los más desarrollados en la región, sin lograr establecer un espacio regional de educación superior.

Palabras clave: educación superior, globalización, América Latina, MERCOSUR.

Mercedes Botto 


\title{
A transnacionalização do ensino supeiror: qualé o papel dos novos regionalismos na difusão dessas ideias? 0 caso do MERCOSUL (1992-2012) em perspectiva comparada
}

\section{RESUMO}

O alvo deste trabalho é contribuir na compreensão da reforma do ensino superior dos anos noventa na América Latina, analisando a influência exercida pelos processos de integração regional, mais exatamente o MERCOSUL. Em que medida ajudou a difundir e pôr em funcionamento as ideias de transnacionalização ou de internacionalização que estavam em debate em nível global? Para responder essa questão, usaremos a comparação da experiência do MERCOSUL com aquela de outros regionalismos, identificando as iniciativas em torno da mobilidade de estudantes e a acreditação de cursos, duas iniciativas que abrangem igualmente os processos de integração analisados. A conclusão atingida sugere que os novos regionalismos avançaram para uma terceira posição: se defender da globalização através de políticas de cooperação solidária e, ao mesmo tempo, procurara conformação de um espaço regional de ensino superior a partir do qual poderiam concorrer globalmente. No entanto, na América do Sul os avanços têm sido reduzidos até hoje na consolidação das capacidades nacionais, promovendo os processos de reforma inconclusos nos países menos desenvolvidos e consolidando as posições estratégicas e dominantes dos países mais desenvolvidos na região, sem conseguir o estabelecimento de um espaço regional de ensino superior.

Palavras chave: ensino superior, globalização, América Latina, MERCOSUL.

\section{The transnationalization of higher education: which role do new regionalisms play in the disemination of these ideas? The case of MERCOSUR (1992-2012) in a compared perspective}

\begin{abstract}
The purpose of this work is to contribute to the understanding of the higher education reform in the 90s in Latin America, analyzing the impact of regional integration processes and more specifically in the MERCOSUR. To what extent did it help to disseminate and launch the ideas of transnationalization or internationalization that were being debated on a global level? To answer this question we will use a comparison of the MERCOSUR experience with other regionalisms, identifying the initiatives related to student mobility and course equivalences, two initiatives that cover the analyzed integration processes. The conclusion we reached suggests that the new regionalisms advanced to a third position: defense against globalization via solidary cooperation policies while trying to create a regional higher education platform to compete at a global level. However, in South America, advancements to date are restricted to the consolidation of national capacities, promoting unfinished reform processes in the less developed countries and consolidating strategic and predominant positions of the most developed ones in the region, without being able to establish a regional higher education platform.
\end{abstract}

Key words: higher education, globalization, Latin America, MERCOSUR.

Recepción: 18/03/13. Aprobación: 27/03/15. 


\section{Introducción}

Los noventa fueron años clave para la reforma de la educación superior (ES) en el mundo. Todos los foros de discusión - a nivel global, regional y nacionalcoincidían sobre la necesidad de introducir cambios en los sistemas nacionales de ES, articulando la enseñanza académica con el mercado laboral y el sector productivo, sacando provecho de las nuevas tecnologías de información y comunicación (TIC).

Con esta idea reaparecía y se actualizaba un viejo debate: el de la internacionalización de la ES como llave de la modernización nacional. Sin embargo, con la globalización económica se enfrentaron dos posiciones aparentemente contradictorias. La primera, sustentada por la UNESCO, que proponía actualizar la vieja fórmula de promover la cooperación horizontal entre los Estados en materia de movilidad estudiantil y redes académicas. La segunda, promovida desde la Organización Mundial de Comercio (OMC), que hablaba de promover el intercambio de servicios educativos con fines de lucro (Knight, 1999; Cottier, 2002; Verger, 2010).

América Latina no estuvo ajena a esta discusión global. A principio de los noventa, las nuevas democracias de la región llevaron adelante un profundo proceso de reforma que puso fin al modelo de "autonomía universitaria", privatizando la oferta, descentralizando la gestión e incorporando mecanismos de control de calidad. La mayoría de los estudios tienden a concentrar su atención en el papel ejercido por el Banco Mundial (BM) en estas reformas y sus impactos a nivel doméstico. Pocos apuntan, en cambio, a analizar la dimensión internacional de esta reforma y a estudiar el impacto de otros actores globales, como la UNESCO y la OMC (López, 2003; Didou, 2006; Gazzola y Didriksson, 2008; Siufi, 2009).

El objetivo de este trabajo es ampliar dicho análisis, focalizando nuestra atención en la influencia que ejercieron los procesos de integración regional y más precisamente el MERCOSUR en estas reformas. En este sentido, y siguiendo los conceptos de Solingen (2012), buscamos responder al siguiente interrogante: ¿en qué medida el MERCOSUR contribuyó a la difusión de las nuevas ideas de transnacionalización de la ES que estaban en boga a nivel global?

Para responder a esta pregunta, usaremos el análisis comparado de la experiencia del MERCOSUR con otros bloques regionales - a saber, la Unión Europea (UE), el Tratado de Libre Comercio de América del Norte (NAFTA, por sus siglas en inglés) y la Asociación de Naciones del Sudeste Asiático (ASEAN, por sus siglas en inglés). Para describir estos casos, recurriremos a fuentes secundarias, mientras que para el MERCOSUR incluimos también algunas entrevistas. ${ }^{1}$

La conclusión a la que llega esta comparación confirma la idea de que estos nuevos regionalismos adoptan una tercera posición: se defienden de la globalización a través de estrategias de cooperación solidaria que buscan posicionar a la región de manera competitiva en el mercado de servicios de ES. El MERCOSUR avanzó en la misma dirección, pero en vez de avanzar a la regionalización de la ES, sus impactos han contribuido a consolidar las capacidades y estrategias nacionales, como la creación de agencias de acreditación en aquellos países que no habían logrado avanzar en ellas bajo el tutelaje del BM.

El trabajo está dividido en tres partes. En la primera se caracteriza el debate global de los años noventa, identificando las principales ideas, los actores y mecanismos de difusión que caracterizaron la antinomia internacionalización vs transnacionalización de la ES. En la segunda parte se analiza de manera comparada las políticas adoptadas por los distintos bloques regionales en materia de acreditación, reconocimiento y movilidad de personas, para concluir con el caso del MERCOSUR y su papel en la reforma

\footnotetext{
${ }^{1}$ En la elaboración de este trabajo, además de fuentes secundarias, se analizaron documentos, informes y entrevistas abiertas a más de 20 académicos, policy makers y técnicos vinculados a los organismos nacionales y regionales de educación superior entre 2008 y 2012 en el marco del proyecto PICT 2008-618.
} 
y los procesos de apertura unilateral que se estaban llevando de manera paralela en la región.

\section{A manera de marco teórico: el rol de los regionalismos en la globalización}

Los noventa fueron años clave para la reforma de la educación superior en el mundo. En este debate intervinieron distintas instituciones como la UNESCO, el BM, la OMC, la Organización para la Cooperación y el Desarrollo Económico (OCDE), el Programa de Naciones Unidas para el Desarrollo (PNUD), la Comisión Europea (CEE), la Organización de Estados Americanos (OEA), el Instituto Internacional para la Educación Superior en América Latina y el Caribe de la UNESCO (IESALC), el Banco Interamericano de Desarrollo (BID), la Agencia Española de Cooperación Internacional (AECI), la Agencia Japonesa para la Cooperación (JICA), la Agencia Canadiense para el Desarrollo (CIDA), la Organización de Estados Iberoamericanos (OIE) y el Convenio Carlos Bello, entre otras (Siufi, 2009: 126).

Dos principales paradigmas o posturas ideológicas caracterizaron la discusión global. Por una parte, estaba la propuesta de transnacionalizar la provisión y regulación de los servicios de ES, quitándole a los Estados una función que hasta entonces les era propia; por otro lado, la idea de internacionalizar las prácticas y recursos entre los países, promoviendo la equidad entre ellos, sin condicionar ni intervenir sobre su legítimo derecho a controlar el acceso y el trato de los proveedores externos (Knigt, 2002; De Wit et al., 2005; Türnnermann, 2006; Didou, 2002b).

Ambas visiones se diferenciaron por distintos elementos. Mientras la transnacionalización hablaba de transformar la educación en un servicio transable y sujeto a las reglas del mercado global, en donde dominan los intereses de las empresas educativas transnacionales, la internacionalización, en cambio consideraba a la ES como un derecho y al conocimiento como un bien colectivo global. De allí la necesidad de promover la cooperación solidaria, entendida como un intercambio horizontal entre pares, basado en el diálogo intercultural, respetuoso de la idiosincrasia e identidad de los países participantes (Lineamientos de la Declaración Mundial sobre la Educación Superior, en Banco Mundial, 1995).

Alrededor de estas dos visiones alternativas de la gobernanza global se articularon las comunidades de políticas (policy networks) lideradas por distintas organizaciones intergubernamentales globales. A la cabeza del paradigma de internacionalización se puso la UNESCO. Su liderazgo no era novedoso como tampoco lo eran sus propuestas. Desde la posguerra hasta los años sesenta y setenta, los esquemas de ayuda internacional se habían basado en acuerdos de intercambio cultural. Con posterioridad, la cooperación para el desarrollo enfatizó sobre las áreas de las ciencias y tecnologías en donde las universidades no tuvieron un papel relevante. La novedad que introducía la globalización fue la visibilidad que adquirió la UNESCO en medio de la controversia ideológica planteada por el neoliberalismo (Cottier, 2002; Zarur, 2008; Siufi, 2009).

El paradigma de transnacionalización estuvo liderado en un primer momento por la OMC (a través de las negociaciones para un Acuerdo general de liberalización de los servicios, GATTS por sus siglas en inglés). Este organismo global argumentaba que, por el hecho de existir proveedores privados en los sistemas nacionales de educación, el arbitraje de los conflictos no podía estar en manos del Estado, que era parte interesada, sino basarse en reglas de control y arbitraje internacional (Cottier, 2002; Verger, 2010). Cuando la OMC habla de liberalizar el comercio de servicios, se refiere a la decisión de los Estados de resignar su derecho al uso de normativas que protejan a los prestadores nacionales vis a vis los extranjeros, permitiéndoles el libre acceso en su territorio y un trato equivalente al que reciben los prestadores nacionales.

La propuesta de la OMC generó una fuerte controversia pública y una escisión entre los países a favor y los países en contra. Aquellos gobiernos que 
promueven la liberalización de la educación superior a través de la OMC y de los acuerdos bilaterales ven en la transnacionalización un gran negocio. ${ }^{2}$ Aquellos que lo miran como una amenaza apuntan a la pérdida de los contenidos nacionales, frente a la probable oferta de programas mediocres de calidad. A pesar del interés y demanda demostrada por países como Suiza, Estados Unidos, Australia, Nueva Zelanda y Japón por liberalizar el comercio internacional; hasta la fecha tan sólo 32 países han contraído algún compromiso concreto en materia de educación postsecundaria, y de ellos sólo dos son latinoamericanos (Türnnermann, 2006; Didou, 2002b). La mayor amenaza no está en el ámbito de la negociación multilateral sino en el marco de las negociaciones bilaterales para la conformación de áreas de libre comercio entre desarrollados y en desarrollo. Es allí donde la capacidad técnica y política de estos últimos se ve menormente disminuida por la asimetría entre las partes (Botto y Peixoto, 2007).

Las diferencias y contrastes entre ambos paradigmas y membrecías se hacen más borrosas cuando nos adentramos en propuestas concretas. Si bien la internacionalización es respetuosa de la diversidad nacional, este principio entra en contradicción cuando la UNESCO y la OCDE promueven políticas de acreditación de calidad que evalúan y estimulan la homogeneización de programas y currículas de acuerdo con propuestas e indicadores preestablecidos, que generalmente, aunque no siempre, están inspirados en las universidades de países desarrollados (Knight, 1999). Lo mismo ocurre con las llamadas agencias facilitadoras de la cooperación intra o interregional, encargadas de la evaluación y aseguramiento de la calidad, en la que parte de sus servicios especializados de intermediación son prestados con fines de lucro, contribuyendo al recrudecimiento del comercio educativo (Didou, 2002b: 14).

Con la globalización económica de los años noventa se produjo un proceso de proliferación de bloques regionales en todo el mundo y de actualización de los viejos procesos de integración hacia los que la literatura especializada llama "nuevos regionalismos". Para algunos autores, se trata de una estrategia ofensiva de los países más ricos para asegurar su hegemonía global (neoliberalismo) por canales alternativos a la vía multilateral de la OMC (Gamble y Payne, 1996, entre otros); para otros, en cambio, constituyen una reacción defensiva frente a las presiones de la OMC y del capital transnacional de abrirse al mundo (Breslin $e t$ al., 2002, entre otros). Una tercera posición, considera que el regionalismo no sólo puede ampliar los márgenes de maniobra de los países en medio de la globalización, sino también favorecer sus participaciones en ésta (Breslin et al., 2002). ${ }^{3}$

La descripción de lo ocurrido con la ES demuestra, como veremos a continuación, que en la mayoría de los casos estamos frente a este tercer escenario. La inclusión de la ES en la agenda de los gobiernos no surgió de la demanda interna, sino que fue una respuesta defensiva a los procesos de transnacionalización promovidos desde la OMC o desde acuerdos bilaterales. Frente a esta amenaza, los países diseñaron e implementaron políticas para posicionarse mejor y de manera más competitiva en el mercado global de los servicios de educación trasfronteriza.

\section{Distintas experiencias regionales frente a las mismas iniciativas La Unión Europea}

La ES pasó a formar parte de las competencias europeas en los años ochenta. La primera intervención

\footnotetext{
${ }^{2}$ A manera de estimación primaria los expertos calculan que el mercado global del subsector de la ES tiene actualmente unos 100 millones de estudiantes en el mundo y que en 2020 tendría 125 millones, de los cuales un 31.5\% cursan en el ámbito privado y el resto en el público, siendo más altos los porcentajes de los alumnos de países en desarrollo. Tomando un costo anual mínimo por estudiante de mil dólares, se estima un mercado de no menos de 31500 millones en el ámbito privado para 2020 (Guarga, en Zarur, 2008: 184). ${ }^{3}$ En este sentido, se puede hablar de una relación simbiótica entre globalización y regionalización en donde la globalización promueve la integración regional, facilitando la inclusión de los países más débiles en la económica global y a su vez, la regionalización promueve la globalización (Breslin et al., 2002).
} 
de la Comunidad Europea (CE) fue en 1987 con el programa de movilidad estudiantil - Plan de Acción de la Comunidad Europea para la Movilidad de Estudiantes Universitarios-, comúnmente conocido como Programa Erasmus. ${ }^{4}$ Su objetivo era construir un sentido de comunidad entre los estudiantes de distintos países europeos a través del intercambio y el aprendizaje de la lengua y cultura del país receptor (Barros de Barros, 2009). La propuesta provino de una red académica de alcance regional - Association des Etats Généraux des Etudiants de l'Europe-, apoyada por los comisionados y gobiernos de los países socialistas de Francia y de España.

Con el tiempo, Erasmus fue ampliando sus alcances y en 1995 fue integrado a un plan educativo más amplio llamado "Sócrates" que, además de la movilidad estudiantil, promovía el intercambio de información y de experiencias comparadas entre distintas instituciones universitarias. Uno de sus logros fue la creación del Sistema Europeo de Transferencia y Acumulación de Créditos (European Credit Transfer System), herramienta fundamental para el reconocimiento mutuo de los programas y conocimientos adquiridos por los estudiantes del Programa Erasmus en su estadía en el exterior. ${ }^{5}$

En 1998 la Unión Europea (UE) hace un salto cualitativo en materia de ES, dejando atrás las políticas de internacionalización a secas para lanzarse a una estrategia de transnacionalización con la que competir con el resto de las potencias mundiales y posicionarse mejor en el mercado de la educación transnacional. Con este propósito, los ministros de educación de Alemania, Francia, Gran Bretaña e Italia firman la Declaración de Sorbona, en donde se comprometen a armonizar la arquitectura del sistema europeo de educación superior y crear un Espacio Europeo de Educación Superior (EEES). ${ }^{6} \mathrm{Un}$ año más tarde, se sumarían los 29 países del bloque y con ello se pone en marcha lo que comúnmente se conoce como Proceso de Bolonia.

Entre los cambios sustantivos que introdujo el Proceso de Bolonia se destacan la estandarización del sistema de acreditación de títulos y la adaptación curricular a un modelo convergente. Para la estandarización de créditos, la CE obligó a las universidades a adoptar un sistema de titulaciones fácilmente legible y comparable que, si bien no suponía una convergencia en términos de contenidos y conocimientos, sí lo hacía en términos de títulos, obligando a todas las carreras a emular la estructura curricular al sistema anglosajón basado en tres ciclos (grado, máster y doctorados), y a eliminar todos los títulos intermedios (como la distinción entre diplomaturas y licenciaturas). Para la adaptación curricular, la CE promovió entre los docentes el uso de nuevas tecnologías en las metodologías de enseñanza, evitando las tradicionales clases magistrales e incluyendo un sistema de calificación permanente para el aprendizaje

\footnotetext{
${ }^{4}$ El Programa Erasmus ofrece a los estudiantes la oportunidad de vivir y cursar sus estudios por un periodo de entre tres meses y un año en otro país europeo. Estos estudios se computan y se reconocen como parte del plan de estudio en la universidad de origen. Desde su creación hasta hoy, se han movilizado a través de este programa alrededor de 2 millones de estudiantes y 140 mil integrantes del personal académico. El programa involucra actualmente a un $90 \%$ de las instituciones de educación superior de la UE, lo que equivale a 3150 universidades en 31 países. El presupuesto anual necesario para la implementación de este programa es de €400 millones de euros (Barros de Barros, 2009).

${ }^{5}$ Su puesta en marcha se dividió en tres fases. En la primera (1995-1999) se administraron unos 200 proyectos de cooperación transnacional entre universidades cuyo objetivo era generar redes de contacto entre departamentos a través de la movilidad de sus estudiantes. En la segunda fase (2000-2006) se aumentó el presupuesto a €1 850 millones y la cobertura del programa, totalizando ocho áreas o disciplinas. Se puso más énfasis en el intercambio del personal docente, la creación de un sistema de reconocimiento mutuo de créditos que permitiera tomar cursos similares en países distintos, en el desarrollo de una currícula transnacional y en la conformación de redes temáticas pan-europeas. Actualmente, el plan Sócrates se encuentra en su tercera fase (2007-2013), denominada LLL (Lifelong Learning). ${ }^{6}$ Este espacio regional buscaba homogeneizar los sistemas nacionales para evitar el éxodo de estudiantes europeos a los Estados Unidos y Japón y competir en mejores condiciones por la llegada de extranjeros. La propuesta era la de crear la "economía del conocimiento más competitiva y dinámica del mundo antes del año 2010, capaz de asegurar un crecimiento económico duradero acompañado por una mejora cuantitativa y cualitativa del empleo y una mayor cohesión social” (Declaración de Lisboa, en Parlamento Europeo, 2010).
} 
en el que la evaluación se hacía de manera continua y donde se privilegiaba la enseñanza práctica. También se avanzó sobre una reforma financiera, que recapitalizaba la universidad, promoviendo la diversificación de fuentes de financiamiento (cuotas para estudiantes, inversiones de empresas privadas) y que fueran éstas, y no los Estados, los responsables de su propia financiación.

A pesar de los cuestionamientos y reacciones en contra que generaron estas iniciativas en asociaciones de estudiantes, docentes y profesionales de países latinos, ${ }^{7}$ el proceso de Bolonia fue exitoso tanto en países del bloque como en extracomunitarios. Una de las razones de este apoyo se encuentra en la distribución de beneficios y recursos materiales que puso a disposición la CE a través de redes de cooperación, programas de apoyo para la creación de consorcios internacionales entre universidades de la región y con otros bloques. A través de estos programas, la UE buscaría no sólo transferir su modelo de ES al mundo en desarrollo sino también armonizar las normativas de otros bloques a las propias, asegurando la inclusión de nuevos países a su propia área educativa y posicionándose mejor en la carrera por la competencia global (Bianculli, 2013). ${ }^{8}$

En cuanto a los resultados, la implementación del proceso de Bolonia no ha sido homogénea en todos los países; en algunos se ha avanzado más que en otros. España, por ejemplo, es un caso en el que el proceso fue tardío pero exitoso. En 2007 introdujo reformas en la ley orgánica universitaria obligando a las universidades a compatibilizar el propio sistema de clasificación de la enseñanza superior con el europeo y de aprobar los planes de estudio por la Agencia Nacional de Evaluación de la Calidad y la Acreditación (ANECA). En el resto de los países mediterráneos - Italia, Francia y Grecia-, aun cuando haya sido aprobada la reforma, su implementación sigue inconclusa.

\section{El Acuerdo de Libre Comercio de América del Norte (TLCAN/NAFTA)}

A diferencia de la UE, en el NAFTA (TLCAN, por sus siglas en inglés) la educación fue considerada desde un comienzo como un bien transable y por ende, sujeta al proceso de desregulación normativa del acuerdo constitutivo, a través de los artículos 11 y 12 referidos a las inversiones y los servicios, respectivamente (Arriaga, 1999; Aboites, 2004). ${ }^{9}$ Sendas normas buscaban promover la inversión y la provisión de servicios por parte de los privados en lo que ahora constituía un mercado de producción y consumo ampliado. El interés de las empresas e instituciones educativas estuvo orientado más a los servicios transfronterizos - a través de la provisión de carreras cortas, de carácter técnico y orientado a satisfacer los requerimientos de los conglomerados industriales que a otro tipo o modalidad de inversión.

Con el tiempo, los gobiernos del bloque fueron incluyendo una serie de iniciativas que buscaron detener la invasión descontrolada de servicios de baja calidad a través de la coordinación de medidas orientadas a la internacionalización de la agenda de ES. Lo interesante de remarcar es que esta agenda de cooperación institucional vino de la mano de actores privados con proyección regional, como la American

\footnotetext{
${ }^{7}$ Las principales críticas se referían a su espíritu "mercantilista" que obliga a las universidades a ajustarse a las necesidades del mercado laboral; someter la docencia y la investigación a las prioridades temáticas de las empresas que las financiaban y a la lotización de la educación al subir las cuotas estudiantiles (especialmente en posgrados). Otros, en cambio, lo acusan de ser un proceso antidemocrático, en donde el sector más consultado fue el privado de alcance regional, a través de la Mesa Redonda de los Industriales Europeos (ERT) en el ámbito de la Comisión Europea (Sanz, 2006).

${ }^{8}$ En el caso concreto del MERCOSUR, la UE intentó que los países miembros adoptaran su sistema de créditos para garantizar luego la movilidad entre sistemas equivalentes (Hermo, 2006).

${ }^{9} \mathrm{Si}$ bien el gobierno mexicano logró establecer algunas restricciones a las inversiones extranjeras en materia de educación durante las negociaciones con sus pares; una vez sellado el acuerdo, fue el propio gobierno el que introdujo cambios sustantivos en la Constitución de México (art. 3) que garantizaba el derecho a la educación; y en la ley de inversiones extranjeras y de educación general, para ajustarse a la lógica pro-mercado (Aboites, 2004).
} 
Council on Education (por la parte de Estados Unidos), el Grupo Condumex (por el lado mexicano), la Northern Telecom y la Max Bell Coorporation (por la parte canadiense). Este grupo de empresas promovió, desde antes de la puesta en marcha del NAFTA, una serie de reuniones trinacionales en las que representantes gubernamentales y de la comunidad académica se sentaron a definir sus objetivos. El espíritu de esta iniciativa quedaría plasmado en su documento original que señalaba que "la Educación Superior debía ser un instrumento para mejorar la competitividad productiva del bloque adaptando la fuerza de trabajo y la investigación aplicada a las necesidades del mercado global" (Boyte y Hollander 1999). ${ }^{10}$

No obstante estas manifestaciones de protestas, ${ }^{11}$ la Declaración de Wingspread se logró plasmar en dos programas de movilidad académica trasnacional. La primera fue la Regional Academic Mobility Program (RAMP), que consistía en una prueba piloto de becas para estudiantes financiada exclusivamente por el Fund for the Improvement of Post-Secondary Education (FIPSE) del Departamento de Educación de los Estados Unidos. La cooperación institucional tomaría la forma de consorcios trinacionales, compuestos por entre seis y nueve universidades, distribuidas de manera equitativa entre los tres países del bloque; en la que cada universidad se comprometía a aceptar estudiantes de los otros dos países sin exigir el pago de una matrícula adicional. En el primer año se dedicarían a establecer el entendimiento y un acuerdo entre las instituciones del consorcio, y a partir del segundo año comenzarían los intercambios de estudiantes y académicos. De esta manera, el programa no sólo buscaba promover el intercambio estudiantil sino también innovación y cambios curriculares en las universidades que formaban parte del consorcio.

Transcurridos los tres años de esta prueba piloto, los gobiernos decidieron lanzar una segunda iniciativa, el Programa de Movilidad Norteamericana (NAMP, por sus siglas en inglés) que actualmente se conoce como Program for North American Mobility in Higher Education. En esta segunda fase se ampliaron a nueve las disciplinas que inicialmente habían estado restringidas a las carreras de ingeniería, negocios y estudios ambientales. También los gobiernos de Canadá y México cambiaron su actitud, involucrándose más activamente en su puesta en marcha, tanto a través de aportes financieros como por una administración conjunta entre el FIPSE, el Human Resources Development Canada (HRDC) y por la Dirección de Desarrollo Universitario de la Secretaría de Educación Pública (SEP) de México. A partir de 2001 las contribuciones de los gobiernos de México y Canadá llegaron incluso a superar las de Estados Unidos.

Al igual que en la UE, la permanencia en el tiempo de estas políticas de movilidad obligaron a introducir cambios en el sistema de acreditación de las carreras. Sin embargo, en este caso, el NAFTA no buscó intervenir en la organización y estructura de los sistemas nacionales sino crear instituciones de acreditación y evaluación de títulos allí donde no existían, como era el caso de México. En el resto de los países del bloque, la acreditación era obligatoria y preexistió al NAFTA. En ambos países la acreditación de títulos y grados requiere la presentación de exámenes para obtener la licencia para el ejercicio profesional una vez que ya se obtuvo el título. Este proceso de evaluación y acreditación lo hacen las asociaciones y

\footnotetext{
${ }^{10}$ La declaración de Wingspread hablaba de cinco objetivos principales: modernizar la educación superior eliminando "obstáculos y reduciendo barreras para mejorar la colaboración trilateral en el campo", lo que podía ser leído como privatización; promover la internacionalización universitaria a través de programas de movilidad estudiantil, mejorar la calidad académica a partir de la colaboración entre instituciones académicas, las autoridades públicas, sectores de negocios y otras organizaciones que tengan interés en la ES; aumentar la eficiencia de las instituciones; favorecer el uso de las tecnologías de difusión, tales como el aprendizaje a distancia y la comunicación por video interactivo, como apoyo a las iniciativas anteriores.

${ }^{11}$ Las protestas y movilizaciones en contra fueron organizadas por sindicatos docentes de los tres países del bloque que formaron una Coalición de Sindicatos para la Defensa de la Educación Pública que consideraba a la educación como un derecho social fundamental garantizado y controlada por los Estados nacionales (Leahy, 2007).
} 
colegios de profesionales a través de sus colegios de acreditación o certificación.

Para concluir, si bien no hay una cuantificación de los resultados de este proceso en términos numéricos - sobre todo en lo que refiere a enseñanza a distancia-, la literatura habla de profundos cambios de índole cualitativo en la universidad mexicana que incorporó en su sistema universitario elementos propios del modelo norteamericano. En primer lugar, la participación del sector educativo privado nacional y extranjero en una conducción tripartita que reemplazó el principio de "autonomía universitaria". ${ }^{12}$ En segundo lugar, la inclusión de un sistema de evaluación y acreditación de carreras de grado y de posgrado. Sin embargo, en el caso mexicano, el sistema no estaría administrado por la sociedad civil, como ocurre en el resto del bloque, sino por el propio Estado, quien sería el encargado de dar fe pública de la calidad y confiabilidad de un profesional o posgraduado, aunque de manera más laxa que en los países del norte (Didou, 2002a).

\section{La Asociación de Naciones del Sudeste Asiático (ANSA/ASEAN)}

En la Association of Southest Asian Nations (ASEAN, por sus siglas en inglés), la propuesta de crear un espacio regional de ES proviene de las universidades públicas de los países más influyentes y poderosos de la región y de organizaciones internacionales, que buscan incluir un número mayor de redes, intercambiando conocimiento, experiencias y capacidades. Se reconoce como antecedente, durante el liderazgo regional de la UNESCO en los años sesenta y setenta, la creación de la Agencia Intergubernamental SEAMEO (su nombre en inglés) y la red RIHED (siglas en inglés) las que finalmente, en 1985 se alinean bajo el nombre de SEAMEO-RIHED, para promover la coordinación y cooperación intergubernamental en temas vinculados con la política, planeamiento y administración de la educación superior.

Los primeros pasos que da la SEAMEO-RIHED en favor de la creación de un espacio regional se orientan hacia una política regional de acreditación de la calidad. En un principio, la evaluación y la acreditación fue vista como un requisito interno de las propias universidades para mejorar sus prácticas nacionales; mas con el tiempo y a consecuencia de la implementación de los programas de movilidad estudiantil, la evaluación se convirtió en un requisito externo para atraer nuevos estudiantes de la región y del exterior.

En 2000, la Red Universitaria del ASEAN (AUN, por sus siglas en inglés) organizó la Quality Assurance Alliance (AUN-QA) formada por un grupo de Oficiales de Calidad (Chief Quality Officers) elegidos por las propias universidades para desarrollar, a manera de punto focal, talleres de discusión y de difusión de buenas prácticas que promovieran la armonización de los estándares educativos en la región (APEID/UNESCO, 2006). La principal diferencia entre la AUN-QA y el sistema impulsado por el SEAMEO-RIHED, radica en que el primero apunta a promover estos criterios a nivel de las instituciones de ES mientras que el segundo lo hace a nivel de las agencias nacionales de evaluación de calidad (Aphijanyatham, 2010).

Fue entonces cuando las universidades primero, y los gobiernos después, crearon redes encargadas de difundir y compartir las buenas prácticas regionales y promover un proceso de armonización voluntaria de los estándares de calificación en el nivel regional. En 2008 la SEAMEO-RIHED (en colaboración con Malaysian Qualifications Agency) creó una red de evaluación de calidad del ASEAN que se llamó AQAN (ASEAN Quality Assurance Network). La primera de estas

\footnotetext{
${ }^{12}$ Esta forma de coordinación tripartita (empresarial-gubernamental-universitaria) serpentea en todos los niveles de la ES, desde instituciones concretas (consejo directivo de las universidades tecnológicas) hasta el ámbito nacional (Coordinación Nacional para la Planeamiento de la Educación Superior, CONPLES), pasando por el impulso a la vinculación entre universidades y empresas nacionales y extranjeras, los convenios de formación de personal para las empresas, el uso de laboratorios y personal universitarios en proyectos para la industria y el surgimiento de instituciones universitarias (fundaciones universitarias) (Aboites, 2004: 60).
} 
redes universitarias se encargaría de evaluar a las universidades, de administrar distintos programas de cooperación (intercambios, becas e investigación) y de gestionar la información. La secretaría de esa red se ubicó en la Universidad Chulangkron de Bangkok. La segunda red regional se encargaría del manejo de la Universidad Virtual del ASEAN y de poner en marcha el primer programa de estudios del ASEAN. En este caso, el papel protagónico lo tuvo la Universidad de Manila.

En cuanto a la movilidad estudiantil, los avances fueron aún menores. Lanzado en 2008 como prueba piloto, estuvo en un principio limitado a tan sólo tres países: Malasia, Indonesia y Tailandia (M-I-T) ${ }^{13}$ Este programa estuvo dirigido por las agencias nacionales de educación superior de cada uno de los tres países y por el SEAMEO-RIHED, y se restringió a sólo cinco disciplinas a nivel de grado consideradas de interés regional: Agricultura, Idiomas y Cultura, Hospitalidad y Turismo, Negocios Internacionales y Ciencias y Tecnologías de los Alimentos. En este sentido, siguiendo a Aphijanyatham (2010), el M-I-T debe ser entendido como una de las primeras iniciativas que apuntan a establecer una infraestructura que ayude a desarrollar una fuerza de trabajo con experiencia intercultural y que responda a las necesidades individuales de los países de la región. Sin embargo, muchas son las asignaturas pendientes, como la extensión hacia el resto de los países y la construcción de un sistema de transferencia de créditos propio, ya que el usado hasta ahora - el University Mobility in Asia and the Pacific Credit Transfer System (UCTS) - se copió del europeo. En esta dirección, el SEAMEORIHED lanzó en 2010 un nuevo proyecto piloto que involucra la movilidad estudiantil en todo el sudeste asiático.

Por último, el SEAMEO-RIHED ha venido trabajando con el objetivo de crear una Área de Educación
Superior del Sudeste Asiático (Southeast Asian Higher Education Area; SEA-HEA), semejante al europeo, y que involucraría unas 10 mil instituciones de educación superior y unos 41 millones de estudiantes. Sin embargo, a diferencia del proceso de Bolonia, en este caso se buscó armonizar los estándares nacionales en lugar de imponer un estándar "superior". Mientras el concepto de estandarización apela a la suscripción a un único esquema, la idea de armonización se apoya en la búsqueda de la comparabilidad entre los distintos sistemas nacionales, preservando la identidad y la diversidad cultural de cada país (como se refleja en cada sistema educativo).

En cuanto a sus resultados, hasta ahora se reducen al intercambio de información y de buenas prácticas entre universidades en materia de planes y sistemas de evaluación. Los avances en las otras dos dimensiones son reducidos, a consecuencia de la ausencia de recursos e incentivos materiales y de actores privados, como ocurre en la UE y el NAFTA, respectivamente. En cuanto a los beneficiarios de estas reformas, sobresalen los procesos de transferencia de información y de experiencia de las universidades de los países más desarrollados hacia los menos desarrollados. Sin embargo, no hay que olvidar que se trata de un proceso voluntario y atado a las capacidades e intereses de las universidades y Estados nacionales para sumarse a estas redes.

$\mathrm{Al}$ igual que el resto de los bloques regionales, el MERCOSUR emprendió políticas vinculadas con la acreditación de carreras y la movilidad estudiantil. Sin embargo, como veremos en esta parte, en vez de contribuir a crear un espacio regional, los alcances hasta el momento ayudaron a fortalecer las capacidades e intereses nacionales. A continuación analizamos ¿cuándo aparece en la agenda de los gobiernos?, ¿cómo se proponen alcanzarlo? y ¿qué resultados se obtuvieron hasta el momento?

\footnotetext{
${ }^{13}$ Dicha iniciativa comenzó como parte del Marco para la Integración Regional en Educación Superior del Sudeste Asiático (Framework for Regional Integration in Higher Education in Southeast Asia), auspiciado por el Consejo del SEAMEO en la 43a Conferencia del Consejo, realizada en 2008 en Kuala Lumpur.
} 


\section{Las peculiaridades del MERCOSUR: el diseño y la implementación de una política regional inconclusa}

En este continente la reforma de la ES antecede a los nuevos regionalismos. Se inicia a principios de los noventa de manera unilateral como parte de las negociaciones de los gobiernos nacionales con el Banco Mundial (BM) para las reformas de ajuste estructural.

La propuesta del BM pone fin al modelo de "autonomía universitaria" que había estado vigente desde los años veinte y que estaba basado en tres principios: educación pública, gratuidad en el acceso, estructura de gestión corporativa y financiamiento estatal. Este sistema había sido funcional a la creación de la sociedad de masas, pero en los años sesenta comenzó a dar señales de agotamiento, fruto del crecimiento de la población y la restricción de presupuesto e inversión pública. Los gobiernos que sucedieron, en su mayoría autoritarios, ensayaron distintas soluciones, como la apertura a la oferta privada en Brasil o política de cupos en Argentina; sin resolver el problema de fondo, terminaron por complicar el panorama y sus soluciones (Mollis, 2003; Gentili y Levy, 2005).

Recién en los años noventa, precisamente en 1993, se avanza en una reforma estructural del sistema apuntando a resolver el problema de baja calidad que era, según el BM común a toda la región y al subdesarrollo. ${ }^{14}$ En sintonía con su ideología neoliberal, la receta del BM apuntó en tres direcciones: abrir la oferta a los proveedores privados y diversificar las fuentes de las instituciones públicas; descentralizar la gestión dándole más autonomía y poder a las instituciones subnacionales para administrar sus propios presupuestos, contratar y despedir al personal y, por último, controlar la calidad de la educación a través de agencias para la evaluación y acreditación de la oferta a nivel de grado y posgrado, que serían autónomas del gobierno de turno, aunque financiadas por el Estado (López, 2003; García de Fanelli, 2010; Suasnábar y Rovelli, 2011; Rodríguez, 2003).

Los avances de estas políticas fueron dispares: la que más avanzó fue la de abrir la oferta educativa a proveedores privados de alcance nacional; mientras que en el resto de las políticas los resultados variaron de país en país. Estas diferencias en las modalidades y timing de las reformas, demuestran que a pesar de la existencia de condicionalidades atadas a los préstamos del BM, los gobiernos nacionales mantuvieron espacios de autonomía y decisión para introducir cambios (Chiroleu, 1999; Chiroleu et al., 2001; Solanas, 2009; Ballerini, 2013).

$\mathrm{Si}$ bien estas reformas unilaterales introdujeron importantes cambios a nivel de los actores domésticos; no lograron modificar el patrón de cooperación internacional que venía caracterizando a la ES en América Latina desde los años sesenta y setenta, salvo por el aumento del flujo y la diversificación de destinos ${ }^{15}$ pero se mantuvo básicamente como una cooperación solidaria de carácter bilateral financiada por Estados Unidos, Alemania, Gran Bretaña, España y Francia, para la movilidad de estudiantes, intercambio de profesores y adquisición de equipos (García, 2003; Didou, 2002b).

El giro hacia la transnacionalización debió esperar a otro actor exógeno - la OMC- y al avance de las negociaciones del GATTS que tuvo lugar en Cancún en 2003. En términos de contenido, la propuesta del GATTS era ideológicamente compatible y complementaria a la que el BM había iniciado con anterioridad. En sintonía con el Consenso de Washington, el BM buscaría desregular la normativa nacional abriendo la competencia a los proveedores

\footnotetext{
${ }^{14} \mathrm{El}$ descenso de la calidad académica no sólo fue el resultado de la expansión de la matrícula y una inadecuada provisión de recursos financieros, sino que estaba agravada por la ineficiencia en la utilización de los recursos existentes y la inequidad que supone la gratuidad de los estudios universitarios por una mayoría de estudiantes provenientes de familias acomodadas; lo cual tiene un efecto negativo en la distribución de los ingresos (Banco Mundial, 1995).

${ }^{15}$ Por lo general, la oferta se circunscribió a disciplinas sin riesgo social, a carreras donde la oferta nacional era escasa (salud, ingeniería, tecnológica y agronomía) y/o a cursos de MBA donde la oferta era mixta a través de convenios con co-titulación.
} 
nacionales; para luego ampliar la apertura a la oferta transnacional y centralizar la regulación a través de normativas multilaterales.

En un primer momento, el rechazo de gobiernos sudamericanos al GATTS fue contundente y en muchos casos contó con el apoyo de redes de universidades públicas y privadas que se unieron para evitar el acceso de proveedores externos y defender la educación "como un bien social y de responsabilidad pública, cuya internacionalización y cooperación internacional debe basarse en valores académicos y culturales" (Zarur, 2008: 185). ${ }^{16}$ Sin embargo, los preceptos del GATTS avanzaron a través de los acuerdos bilaterales de libre comercio que países como Chile (2003), Colombia (2004), Costa Rica (2004) y Perú (2004), firmaron con países/regiones desarrolladas.

Los países del MERCOSUR no fueron una excepción. La propuesta que más avanzó fue la de la UE en 1994 de alcanzar un acuerdo de libre comercio entre ambas regiones. El interés del MERCOSUR por obtener el acceso de sus productos agrícolas y la cooperación financiera y técnica de la UE, llevó a los países a firmar un compromiso para la liberalización del comercio de servicios intrazona en un plazo no mayor a diez años (Protocolo de Montevideo, 2007). Sin embargo, los gobiernos no avanzaron en su implementación, a medida que su motivación (la negociación con la UE) fue perdiendo vigor (Botto y Peixoto, 2007).

$\mathrm{El}$ avance del comercio transnacional fue paulatino. En un primer momento, la oferta de servicios provino del sector privado externo y adoptó la forma de iniciativas individuales o de redes (consorcios entre universidades o con empresas) que ofrecían posgrados y licenciaturas en carreras cortas vinculadas con la formación profesional. Con posterioridad, los gobiernos empezaron a promover la inversión externa privada y la venta de servicios educativos hacia otros países de la región, aprovechando la cercanía. Las modalidades variaron desde la instalación de oficinas de enlace en otros países - como hizo la Universidad de Chile en Washington, o la Universidad Andina Simón Bolívar en Bogotá-, hasta la convocatoria de capitales a sus propios países con proyectos de coinversión - como la Universidad de las Américas en Uruguay. Los gobiernos, por su parte, buscaron promocionar de manera sistemática sus instituciones académicas, creando comités de servicios educativos, como ocurre con las embajadas de Chile en el exterior desde 2002 (González, 2003).

Los resultados de este fenómeno de transnacionalización son inciertos porque es muy dificil obtener datos precisos sobre los flujos de este tipo de comercio. Los estudiosos tienden a considerar que su impacto ha sido fuerte pero circunscripto. Sin embargo, existen factores estructurales en la región que la vuelven vulnerable y contribuyen a potenciar una invasión de oferta de origen incierto y de baja calidad. En primer lugar, la gran diversidad normativa y regulatoria que existía entre países limítrofes sobre condiciones y requisitos de funcionamiento de instituciones educativas extranjeras (Zarur, 2004). ${ }^{17}$ En segundo lugar, las fuertes asimetrías que separan a los países en materia de información y de control de la propia oferta académica. Esta situación se agravaba por la ausencia en muchos países de una agencia de acreditación de calidad de carreras de grado y de posgrado y por una puesta en marcha inconclusa o disfuncional en otros (Chiroleu, 1999; Hermo, 2006; Perrota, 2011)..18

\footnotetext{
${ }^{16}$ Expresiones de este acompañamiento son la Carta de Porto Alegre, acordada en el marco de la III Reunión de Rectores de las Universidades Iberoamericanas, en abril del 2002; la Declaración de Bogotá del CXI Consejo Nacional de Rectores de la Asociación Colombiana de Universidades (ASCUN) de 2004 y el Grupo Montevideo de Universidades del MERCOSUR.

${ }^{17}$ En este sentido encontramos gran diversidad de normativas en la región: hay países que prohíben la apertura (República Dominicana), otros donde no está legislado (Chile, Perú, América Central), otros en donde está sometido al mismo régimen que las privadas (Bolivia); otros donde los proveedores foráneos están sujetos a un régimen especial (Argentina, decreto 276/99), o países como Colombia donde están obligadas a asociarse a instituciones nacionales de educación superior o a funcionar sin ánimo de lucro (Zarur, 2004). ${ }^{18} \mathrm{Si}$ bien este objetivo formaba parte de las reformas propiciadas por el BM, sólo se logaron instalar en algunos países como Argentina, Brasil, México, Chile, Colombia, Costa Rica y Cuba. De todos ellos, Brasil era el único que tenía alguna experiencia previa en materia
} 


\section{Un diseño inspirado en la experiencia de la UE}

La preocupación por esta situación de vulnerabilidad estuvo instalada en el MERCOSUR desde el inicio del proceso. Aun antes de tener un espacio institucional donde reunirse, los ministros de educación de los cuatro países miembros (sumándose los de Chile y Bolivia) coincidieron sobre la necesidad de priorizar la agenda de ES. ${ }^{19}$ Si bien se hablaba de promover la cooperación solidaria (internacionalización) a través de la movilidad estudiantil y la cooperación interinstitucional, también se incluyeron otras iniciativas como la de la acreditación regional en vista a formar un espacio regional de educación superior. ${ }^{20}$

Los programas de acreditación fueron dos y se aplicaron de manera progresiva. El primero llamado Mecanismo Experimental de Acreditación (MEXA) incluyó a las carreras de Medicina, Ingeniería y Agronomía entre 2002 y 2006. El segundo, Sistema de Acreditación de Carreras Universitarias para el reconocimiento de las respectivas titulaciones en el MERCOSUR y sus Estados Asociados (ARCU-SUR), se inició en 2007 con vistas a concluirse en 2012. Inicialmente el ARCU-SUR incluyó a las carreras de Veterinaria, Enfermería, Odontología y Arquitectura; pero se diseñó con la idea de convertirse en un mecanismo permanente, vinculante y aplicable a todos los niveles de ES (grado, posgrado y tecnicaturas).
A diferencia de la UE, la acreditación en el MERCOSUR buscó respetar las autonomías nacionales, armonizando los estándares de calidad nacionales a uno regional — sello MERCOSUR - creado por expertos regionales con base en el perfil curricular, la calidad de los docentes y el número de egresados, entre otras consideraciones. No se trataba de ajustar la normativa a un modelo específico, sino de un proceso de intercambio y diálogo a través de reuniones entre académicos, técnicos y funcionarios, en nivel nacional y regional, para promover redes de confianza y de reconocimiento mutuo entre las distintas instituciones de la región. ${ }^{21} \mathrm{El}$ costo sería afrontado por cada institución académica ya que se esperaba que fueran las universidades las que tuvieran interés en adquirir el sello MERCOSUR para poder competir en mejores condiciones en el mercado regional y atraer más estudiantes y recursos financieros y de cooperación técnica (Botto, 2013). ${ }^{22}$

En materia de movilidad académica también se diseñaron dos propuestas consecutivas e íntimamente vinculadas con la política de acreditación. La primera se llamó Programa de Movilidad Académica Regional de Cursos Acreditados (MARCA) y la segunda Programa Movilidad MERCOSUR (PMM). El objetivo de ambas iniciativas era generar redes de cooperación inter-institucional que contribuyeran a mejorar la calidad de la enseñanza, promoviera la

de evaluación - Coordenação para o Aprofundamento da Educacão Superior (CAPES) creada en los sesenta/setenta para carreras de posgrado. La reforma del BM vino a ampliar sus alcances a las carreras de grado a la vez que un sistema de acreditación (oficial) conformado por dos instituciones - la Secretaría de ES del Ministerio de Educación (SESU) y el Instituto Nacional de Investigaciones Educativas (INEP) - ; además de una evaluación externa y un examen nacional de calidad llamado "Provao" (Hermo, 2006).

${ }^{19} \mathrm{La}$ agenda de los ministros estaba dividida en los cuatro niveles educativos (primario, secundario, técnico y superior). Sin embargo, mientras en los primeros niveles la lógica que primó fue la de promover el intercambio de información y el reconocimiento mutuo, en el nivel de la ES se avanzó hacia la coordinación de políticas regionales (entrevista a asesor brasileño del ministerio de educación, FLACSO, octubre 2010).

${ }^{20}$ En la segunda RME se acordó “incrementar el intercambio académico-técnico-científico en el nivel de enseñanza superior, posibilitando una mayor movilidad de docentes, alumnos, investigadores y técnicos [...para ello] se promoverá la creación y perfeccionamiento de los mecanismos de equiparación y reconocimiento de estudios, títulos y diplomas, entre los países integrantes del MERCOSUR" (Art. $2^{\circ}$, RME, Acta 02/92, el énfasis es nuestro).

${ }^{21}$ Entrevista a funcionaria de la CONEAU, Buenos aires, junio 2013.

${ }^{22}$ En algunos casos funcionó así y la propuesta fue aceptada por las universidades nacionales que, celosas de su autonomía, se resistían a ser auditadas y evaluadas por organismos nacionales como la CONEAU. Un caso paradigmático fue el de la carrera de Agronomía de la Universidad de Buenos Aires, que finalmente aceptó el proceso de acreditación nacional pero no se presentó al proceso de acreditación regional por temor a quedar excluidos de los beneficios que derivarían de las convocatorias regionales (entrevista a funcionario de la CONEAU, junio 2014). 
investigación conjunta, ayudaran a adecuar la gestión y la infraestructura institucional a los requerimientos de los estudiantes extranjeros y, por último, que promovieran el conocimiento recíproco de lenguas y culturas. Los alcances de esta política estuvieron recortados al universo de carreras acreditadas por MEXA y ARCU-SUR, respectivamente y condicionados al financiamiento y apoyo técnico de la UE; ante la ausencia de fondos regionales, se pensó que correría a cuenta de los Estados nacionales. En los hechos, esta política estuvo desde un comienzo fuertemente atada y condicionada. ${ }^{23}$

El tercer eje - cooperación inter-institucionalfue el que menos avanzó por falta de financiamiento. Esta constatación llevó a los gobiernos en 2004 a crear un Fondo Educativo del MERCOSUR (FEM) formado principalmente con los aportes de los Estados, siguiendo una lógica distributiva en la que los países más pequeños son los que más reciben y los que menos aportan.

\section{Una implementación orientada a profundizar las reformas unilaterales}

La acreditación regional fue la política que más avanzó en términos de duración y cobertura, proyectando sus alcances a la UNASUR en la actualidad. Distintas razones explican este éxito. Por una parte, no requería como en el resto de las iniciativas de grandes financiamientos, ya que se trataba más bien de un proceso de armonización normativa donde la principal inversión corría por cuenta de los beneficiados de los procesos y/o en desmedro de los Estados nacionales. Por la otra, esta iniciativa gozó desde un principio de un activo liderazgo político, por parte del gobierno argentino que, a través de su exitosa experiencia nacional de acreditación, buscó proyectarse como nuevo líder regional en la cooperación Sur-Sur. También fue clave el liderazgo que asumieron los funcionarios técnicos de las agencias de acreditación nacional en su capacidad para conformar redes de políticas que neutralizaron en muchos casos las resistencias provenientes de funcionarios y lobbies nacionales, que veían al proceso desde su interés nacionalista.

No obstante ello, los avances de esta reforma no fueron lineales ni incrementales. En su puesta en marcha fue perdiendo impulso en favor de iniciativas bilaterales y algunos de sus aspectos originales clave fueron eliminados, como el cumplimiento de los plazos de ARCU-SUR; la reducción de su cobertura a carreras de grado (eliminando las de posgrado) y la quita del reconocimiento de títulos profesionales (limitándolos tan sólo a los académicos). Todas estas quitas demuestran el progresivo desinterés de los gobiernos, sobre todo de Brasil, por articular la capacitación académica al mercado laboral y en gran medida reflejó el peso que los lobbies internos - cámaras profesionales e instituciones - fueron adquiriendo a medida que las promesas políticas se fueron implementando.

Los resultados de este proceso de acreditación regional no fueron significativos en términos numéricos. ${ }^{24}$ También dejaron en evidencia las grandes asimetrías que separaban a los países del bloque ya que la mayoría de las carreras acreditadas pertenecían a las universidades públicas de renombre de Argentina y de Brasil que, en definitiva, eran los únicos que podían financiar este proceso tan costoso. Para la mayoría de los protagonistas y estudiosos del tema, en cambio, la principal contribución de esta política

\footnotetext{
${ }^{23}$ A manera de ejemplo, el PPM fue elaborado a partir de un diagnóstico realizado por funcionarios regionales (de la CRC-ES) y expertos de la UE, que entre los meses de septiembre y octubre de 2005 visitaron las IES de los cuatro países miembro. En la actualidad (presupuesto 2012), el 75\% corre por cuenta de EU y el resto por los intereses que deja el FEM, (entrevista funcionaria de Asuntos Universitarios del Ministerio de Educación de la Argentina).

${ }^{24}$ Hacia fines de 2012, los números del MEXA + MARCA - ARCUSUR, señalaban un total de 267 carreras acreditadas y un total de 800 alumnos becados para hacer estudios en el exterior. De estas 267 carreras acreditadas en 2012 , 167 eran argentinas, 18 uruguayas y 7 del Paraguay.
} 
regional a la internacionalización de la ES fue de tipo cualitativo y se manifiesta en su capacidad de acopiar la información, en la construcción de reconocimiento mutuo entre sistemas en los que se desconfiaba hasta entonces y los avances en la definición de parámetros de calidad en algunas carreras. ${ }^{25}$ Pero sobre todo, este proceso contribuyó a fortalecer las capacidades técnicas e institucionales nacionales de aquellos países que no habían logrado avanzar en este aspecto de las reformas unilaterales promovidas por el BM. En este sentido, la integración regional a través de la experiencia montada por las agencias nacionales de Argentina (CONEAU), Chile (CNAP) y Brasil (CONAES) ayudó a crear organismos de evaluación y acreditación en países donde - como Uruguay, Paraguay, Bolivia - no existían o no estaban en funcionamiento. ${ }^{26}$ Este patrón de cooperación Sur-Sur se mantiene en la actualidad, bajo una dinámica bilateral liderada por Argentina y se ha ido ampliando a los países de la UNASUR, como Ecuador y Venezuela.

A diferencia de la acreditación, los programas de movilidad estudiantil y de cooperación interinstitucional tuvieron pocos resultados. En primer lugar, porque dependieron de fondos externos, principalmente de la UE cuya continuidad en el tiempo estuvo atada a la buena salud de la negociación birregional. En segundo lugar, porque carecieron de interés y liderazgo regional. Aun cuando existió decisión para crear un Fondo de Educación para el MERCOSUR (FEM), su puesta en marcha se vio obstruido por su principal aportante, Brasil, que en un primer momento postergó el desembolso y con posterioridad el consenso en torno a las prioridades para su uso. ${ }^{27}$ Con el reposicionamiento de Brasil como líder global, su estrategia se ha orientado en los últimos años a invertir en programas bilaterales de cooperación solidaria, financiando la movilidad de estudiantes y profesionales de la región y de la UNASUR en aquellas disciplinas y áreas donde la oferta nacional es escasa o incompleta.

Por último, la iniciativa que mejor ilustra el dilema que actualmente atraviesa la integración regional en América Latina es la de crear un Espacio Regional de Educación Superior (ERES). La propuesta aparece en la agenda regional, de manera tardía, en 2007, cuando ya se conocían experiencias de otros bloques en donde este espacio se instalaba como corolario de un proceso de acreditación y movilidad con vistas a competir por alumnos e inversiones a nivel global. En el MERCOSUR, el ERES se visualizó como un espacio de cooperación tradicional. Entre las propuestas que se hicieron estaba la de Brasil de crear una Universidad de Integración Latinoamericana (UNILA), que por falta de apoyo regional terminó siendo implementada de manera unilateral a través de recursos nacionales. No obstante ello, las discusiones y posicionamiento conjuntos en el marco de las reuniones de ministros de educación del MERCOSUR y de la UNASUR, ha servido a los países y actores de la región como un ámbito de consenso y de posicionamiento regional en favor de la defensa de la educación superior pública y gratuita en los distintos foros de discusión y cooperación global, como son la UNESCO y el IESSP. ${ }^{28}$

\footnotetext{
${ }^{25}$ Entre los acuerdos firmados está el Protocolo de Integración Educativa sobre Reconocimiento de Títulos Universitarios para la Prosecución de Estudios de Posgrado en las Universidades de los países del MERCOSUR, que posibilitaría también el reconocimiento posterior de títulos de posgrado obtenido, y el Protocolo de Integración Educativa para la Formación de Recursos Humanos a nivel de posgrado en los países del MERCOSUR (Acta 02/95 RME).

${ }^{26}$ En el caso de Paraguay se crea una Agencia de Evaluación y Acreditación de la Educación Superior (ANEU); en Uruguay, si bien no se crea un ANA, se organizaron comisiones ad hoc y se entrenaron a los funcionarios para que pudieran participar del proceso de acreditación regional. Bolivia, sin lugar a dudas, es un caso especial, ya que en 1995 crea por ley un sistema nacional de acreditación y medición de calidad educativa (CONAMED) que nunca se había logrado poner en marcha por la oposición de las universidades a esto que consideraban una intromisión.

${ }_{27}$ Entrevista a asesor brasileño, FLACSO Buenos Aires, octubre 2010.

${ }^{28}$ Entrevista a funcionario del Grupo de Universidades de Montevideo, Buenos Aires, agosto 2014.
} 


\section{CONCLUSIONES}

Retomemos entonces la pregunta que dio origen a este artículo: ¿qué rol jugó y juega el MERCOSUR en la difusión de las ideas de internacionalización/ transnacionalización de la ES en América Latina?

La integración regional surgida o actualizada en los años noventa en esta parte del mundo tuvo, al igual que en el resto de los bloques, el objetivo de hacer frente a través de soluciones colectivas a los desafios y oportunidades que planteaba la globalización económica. Sólo que en este contexto regional, como lo sugiere el artículo, los desafíos eran más complejos, ya que a los problema globales se sumaba la crisis y agotamiento de los propios sistemas educativos. A esto se suma la fuerte influencia de actores externos con propuestas y soluciones no siempre compatibles, sino diferentes y hasta contradictorias.

En este sentido, una de las primeras conclusiones a las que contribuye este artículo es demostrar que los procesos de reforma de la ES en América Latina no se han reducido, como parecen sugerir la mayoría de los estudios sobre el tema, a un proceso de reforma unilateral, condicionado por los préstamos del BM, sino representan también un proceso de apertura transnacional, liderado por actores globales y regionales, como la OMC, la UNESCO, la UE y la negociación del ALCA. Si bien en apariencia parecía existir una clara división de trabajo entre estos organismos en relación con el contenido, alcances y mecanismos de difusión de sus propuestas de reforma de ES para el subdesarrollo, en la práctica, el análisis sugiere una continuidad entre la propuesta de desregulación y privatización del BM y el paso subsiguiente de abrir esta oferta a proveedores externos y controlar el comercio a través de regulaciones globales como propone la OMC y la UE.

La segunda conclusión que sugiere el artículo es que los procesos de integración regional tomaron posición en el debate global, proponiendo una visión alternativa a las dos visiones contrapuestas entre la idea de la UNESCO de profundizar la cooperación solidaria manteniendo el control y responsabilidad de la ES en manos de los Estados nacionales, y la de la OMC que proponía desregularla y dejarla liberada a la oferta y demanda de servicios a nivel transnacional. La respuesta de los nuevos regionalismos a esta dicotomía fue la de crear un espacio regional de ES en el que se crearan estrategias regionales de cooperación solidaria (internacionalización) entre los Estados miembros para mejorar la calidad de la oferta, competir globalmente y atraer la demanda interna y externa (transnacionalización).

En el contexto latinoamericano, el MERCOSUR fue el proceso de integración Sur-Sur que más avanzó en la materia. $\mathrm{Al}$ igual que en otras regiones, esta política se orientó hacia tres tipos de programas: la acreditación regional de títulos, la movilidad transnacional de estudiantes y profesores, y la cooperación interinstitucional para la transferencia de recursos. La comparación de los distintos bloques sugiere que si bien se trataba de las mismas políticas, las modalidades y el tipo de bien regional alcanzado varió en cada uno de ellos. En el caso latinoamericano, se priorizó una política de acreditación de títulos que no interviniera sobre las regulaciones nacionales, sino que estableciera un parámetro de comparabilidad de la calidad entre las instituciones que otorgaran un mismo título. Las políticas de movilidad y cooperación, en cambio, no logaron mayores avances por falta de recursos compartidos y estuvieron atadas a las ayudas y financiamientos externos.

En el proceso de implementación, la política de acreditación en el MERCOSUR fue perdiendo el impulso inicial: el liderazgo regional se redujo a uno bilateral; se desarticuló la formación académica de la profesional, en favor del reconocimiento de títulos de grado; se restringió su cobertura universal a un grupo de carreras universitarias. Este artículo señala que algunas de estas restricciones fueron resultado de decisiones originales vinculadas a la decisión de los gobiernos de no inmiscuirse en las autonomías nacionales, evitando la intervención sobre las 
currículas y formas de evaluación nacional; lo anterior dejó la decisión a un acto de voluntarismo de las propias instituciones y redujo la responsabilidad de los Estados más ricos de crear instituciones regionales de financiamiento.

Otras razones, en cambio, son resultado de factores contextuales, como la existencia de profundas asimetrías en materia de infraestructura educativa, de capacidad de gestión, de control de calidad y de estructura económica en la región. Lejos de achicarse con el tiempo, el artículo sugiere que estas diferencias se fueron ampliando, debido también a los cambios en el contexto regional global. El primero fue la proliferación de acuerdos de libre comercio entre los países latinoamericanos y los países desarrollados, que amplió la oferta e innovación de servicios transnacionales en la región, ahora motorizados por ofertas público-privadas. El segundo cambio global se materializó en el surgimiento de los países emergentes y en la proyección de Brasil como líder global, lo que redundó en la quita definitiva de su apoyo a las iniciativas regionales para desplazarla hacia la cooperación bilateral dirigida a Sudáfrica y América Central.

La cuarta y última conclusión se refiere a los resultados obtenidos hasta la fecha. De la comparación de otros casos con el MERCOSUR, el análisis sugiere que la principal contribución del MERCOSUR ha sido la de contener las propuestas de la OMC y de las negociones de ALCA y la UE de transnacionalizar el comercio de servicios educativos y la de crear un espacio de cooperación solidaria desde donde fortalecer la confianza y el reconocimiento mutuo entre países e instituciones transfronterizas.

Sin embargo, a diferencia de los otros bloques, no ha sido hasta ahora capaz de avanzar hacia la creación de un espacio regional de ES para posicionarse como jugador global y atraer la demanda del exterior. Sus avances, en este sentido, se han reducido a fortalecer las capacidades nacionales. En el caso de Brasil, el MERCOSUR le ha permitido conocer e identificar los recursos disponibles para atraerlos y suplir los déficits que su país tiene en materia de profesionales e investigadores, convirtiéndose paulatinamente en uno de los nuevos destinos de la fuga de cerebros en la región. En el caso de Argentina, la experiencia nacional y regional adquirida en el proceso de acreditación, le ha permitido posicionarse en el escenario regional como líder en la cooperación Sur-Sur con países de la UNASUR. En el caso de las economías más pequeñas, el MERCOSUR ha contribuido a la instalación de agencias de acreditación nacional que eran disfuncionales o inexistentes, transfiriendo la experiencia de los países más desarrollados a los menos desarrollados, replicado el modelo de cooperación Norte-Sur ¿De esto se trata la modernización? 


\section{Referencias}

Aboites, H. (2004), "Actores y políticas en la educación superior mexicana: las contradicciones del pacto de modernización empresarial", en M. Molis (comp.), La universidad en América Latina: ¿Reformadas o alteradas? La cosmética del poder financiero, Buenos Aires, CLACSO, pp. 57-86.

APEID/UNESCO (2006), Learning Together for Tomorrow Education for Sustainable Development, Tailandia, UNESCO/Asia and Pacific Regional Bureau for Education (APEID).

Aphijanyatham, Ropharat (2010), East Asian Internationalisation of Higher Education. A key to regional integration, Japón, University of Tsukuba-Center for Research on International Cooperation in Educational Development.

Arriaga Lemus, María de la Luz (1999), "NAFTA and the Trinational Coalition to Defend Public Education", en Social fustice Review, vol. 26, núm. 3, pp. 145-155.

Banco Mundial (1995), La enseñanza superior. Las lecciones derivadas de la experiencia, Washington, D.C. Publicación BM/WB, 99. 115.

Ballerini, V. (2013), "La internacionalización de la educación superior en la Argentina”, Tesis de maestría, Facultad Latinoamericana de Ciencias Sociales (FLACSO), sede Argentina (directora M. Botto).

Barros de Barros, Fabiana (2009), "El Programa Erasmus como propulsor del Espacio Europeo de Educación Superior", en Germán Bernacer (ed.), Casos prácticos para la gestión de la internacionalización en universidades de SAFIRO II-Self Financing Alternatives for International Relations Offices II, Alicante, Universidad de Alicante.

Breslin, S., R. Higgott y B. Rosamond (2002), "Regions in comparative perspective", en S. Breslin, R. Higgott y B. Rosamond (eds.), New regionalism(s) in the global political economy: theories an cases, Londres, Routledge, pp. 1-19.

Bianculli, Andrea (2013), "The effect of trade agendas on regulatory governance: when the EU meets the global south", documento de trabajo, en KFG The Transformative Power of Europe, Working Paper Series, p. 58.
Botto, Mercedes y Peixoto Batista, Juliana (2007), "La incidencia de la academia en las negociaciones de los servicios de salud y educación en la Argentina: desafíos y oportunidades", en Mercedes Botto (coord.), Saber y política en América Latina, Buenos Aires, Prometeo Libros, pp. 247-292.

Botto, Mercedes (2013), "Los regionalismos y la difusión de políticas: la educación superior en el MERCOSUR", en Densidades, núm. 14, http://issuu.com/densidades/ docs/densidades_n_14.

Boyte, Harry y Elizabeth Hollander (1999), Wingspread Declaration on Renewing the Civic Mission of the American Research University, disponible en http:// kdp0143vw6z2dlw63 1ififc5.wpengine.netdna-cdn. com/wp-content/uploads/2009/04/wingspread_ declaration.pdf [consulta: febrero 2013].

Chiroleu, Adriana (1999), El ingreso a la universidad. La experiencia de Argentina y Brasil, Rosario, UNR Editora.

Chiroleu, A., O. Iazetta, C. Voras y C. Diaz (2001), "La política universitaria argentina en los 90s. Los alcances del concepto de autonomía", en Education Policy Analysis Archives, núm.9, (22) junio, pp. 178-184.

Cottier, Thomas (2002), "Trade and human rights: a relationship to discover", en fournal of International Economic Law, Glasgow, UK, Oxford University Press, vol. 5, núm. 1, pp. 111-132.

DeWit, H., I. Jaramillo, J. Gacel-Avila y J. Knight (eds.) (2005), Higher education in Latin America. The international dimensión, Banco Mundial, Washington.

Didou Aupetit, Sylvie (2006), "Internacionalización de la educación superior y provisión transnacional de servicios educativos en América Latina: del voluntarismo a las elecciones estratégicas", Ponencia en el Seminario Internacional IESALC-UNESCO, Conferencia de Rectores, Panamá, 16 al 17 de marzo, pp. 1-19.

Didou Aupetit, Sylvie (2002a), Transnacionalización de la Educación Superior en México, Caracas, UNESGO-IESALC.

Didou Aupetit, Sylvie (2002b), "Globalization, NAFTA and higher education system in Mexico: issues, challenges, 
and reforms", en Educational Sciences: Theory E Practice, vol. 2, núm. 1, pp. 81-92.

Gamble, A. y A. Payne (1996), Regionalism and the world order, Basingstoke, Macmillan.

García Guadilla, C. (2003), "Balance de la década de los '90 y reflexiones sobre las nuevas fuerzas del cambio en la educación superior", en M. Mollis (comp.), Las universidades en América Latina: ¿reformadas o alteradas? La cosmética del poder financiero, Buenos Aires, CLACSO, pp. 17-38.

García Guardilla, C. (2005), "Complejidades de la globalización e internacionalización de la educación superior: interrogantes para América Latina", en Cuadernos CENDES, año 22, núm. 58, enero-abril, pp. 1-22.

García de Fanelli, A. (2010), "Mecanismos de financiamiento de los gobiernos a las universidades latinoamericanas: innovaciones en las últimas décadas", en F. López Segrera y D. Rivarola (comps.), La universidad ante los desafios del Siglo XXI, Paraguay, Quinquenio del Cincuentenario de la Revista Paraguaya de Sociología.

Gazzola, A. y A. Didriksson (2008), "Prefacio", en A. Gazzola y A. Didriksson, Tendencias de la educación superior en América Latina y el Caribe, Caracas, IESALC-UNESCO, pp. 9-20.

Gentili, P. y B. Levy (comps.) (2005), Espacio público y privatización del conocimiento: estudios sobre políticas universitarias en América latina, Buenos Aires, CLACSO.

González, L. (2003), Los nuevos proveedores externos de la educación superior en Chile, Santiago, Digital Observatory for Higher Educiation in Latin america and the Caribbean- IESALC, disponible en www.iesalc.unesco. org.ve [consulta: febrero 2013].

Hermo, J. (2006), "La acreditación regional de carreras en el MERCOSUR: presente y futuro", Tesis de maestría, FLACSO, Argentina.

Knight, J. (1999), "Internationalization of higher education", en J. Knight y H. de Wit (eds.), Quality and internationalization in higher education, París, OECD.

Leahy, A. (2007), “Coalición Trinacional para la Defensa de la Educación Pública en Canadá, Estados Unidos y México. 1993-2007”, http://www.trinationalcoalition. org/docs/trinational\%20history.07.spanish.doc.

López Segrera, F. (2003), "El impacto de la globalización y las políticas educativas en los sistemas de educación superior en América Latina y el Caribe”, en M. Mollis (comp.), Las universidades en América Latina: ¿reformadas o alteradas? La cosmética del poder financiero, Buenos Aires, CLACSO, pp. 39-58.

Mollis, M. (2003), Las universidades en América Latina: ¿reformadas o alteradas?, Buenos Aires, CLACSO.

Parlamento Europeo (2010), The Lisbon Strategy 2000-2010: an analysis and evaluation of the methods used and results achieved, Reporte final, Bruselas, Directorate General for Internal Policies/Policy department a: economic and scientific policy.

Perrotta, Daniela (2011), "La integración regional de la educación superior en el MERCOSUR en el marco de la orientación general del bloque y la tensión entre un modelo solidario y otro competitivo: el caso de los programas regionales de acreditación de carreras de grado (del MEXA al ARCU-SUR, 1998-2008)", Tesis de maestría, FLACSO, Argentina.

Protocolo de Montevideo (2007), Decisión CMC No 13/97, www.mercosur.int/.../Protocolo $\% 20 \mathrm{de} \% 20$ Montevideo\%20rev\%201.ppt.

Rama, C. (2006), La tercera reforma de la educación superior en América Latina, México, Fondo de Cultura Económica.

Siufi, Gabriela (2009), "Cooperación internacional e internacionalización de la educación superior", en Educación Superiory Sociedad, vol. 14, núm. 1, pp. 120- 145.

Rodríguez Gómez, R. (2003) "La educación superior en el mercado", en M. Mollis (comp.), Las universidades en América Latina: ¿'reformadas o alteradas? La cosmética del poder financiero, Buenos Aires, CLACSO, pp. 87-107.

Sanz Fernández, Florentino (2006), "La mercantilización de la educación como escenario mundial del Espacio Europeo de Educación Superior", en Educación XXI, núm. 9, Facultad de Educación, UNED, pp. 57-76. 
Solanas, Facundo (2009), "La ley de educación superior en Argentina, un análisis en términos de reforma de la acción pública", en Revista de Educación Superior, núm. 149, pp.155-170.

Solingen, E. (2012), "Of dominoes and firewalls: the domestic, regional, and global politics of international diffusion", en International Studies Quaterly, num. 56, pp. $631-644$.

Soderbaum, F. (2003), "Introduction: theories of new regionalism", en F. Soderbaum y T. Shaw (eds.), Theories of New Regionalism, Londres, Palgrave Macmillan.

Stubrin, A. (2005), Mecanismo experimental de acreditación en el MERCOSUR + Chile y Bolivia. Elogio de la evolución y acreditación de la educación superior como herramienta de cooperación horizontal para el desarrollo y la integración internacional en bloques regionales, París, UNESCO.

Suasnábar, C. y L. Rovelli (2011), "Políticas universitarias en Argentina: entre los legados modernizadores y la búsqueda de una nueva agenda", en Innovación
Educativa, vol. 11, núm. 57, octubre-diciembre, pp. 2130, México, Instituto Politécnico Nacional.

Tünnermann Bernheim, Carlos (2006), "Internacionalización y transnacionalización de la educación superior. Algunas reflexiones", en Perfiles, núm.76, pp. 76-83.

Verger, A. (2010), WTO/GATS and the global politics of higher education, Nueva York, Routledge.

Zarur Miranda, Xiomara (2008), "Integración regional e internacionalización de la educación superior en América latina y el Caribe", en A. Gazzola y A. Didriksson (eds.), Tendencias de la educación superior en América Latina y el Caribe, Caracas, IESALC-UNESCO, pp. 179-240.

Zarur Miranda, Xiomara (2004), Los nuevos proveedores externos de educación superior en Colombia, Bogotá, Digital Observatory for Higher Educiation in Latin america and the Caribbean- IESALC, disponible en www.iesalc. unesco.org.ve [consulta: febrero 2013].

Cómo citar este artículo:

Botto, Mercedes (2015), “La transnacionalización de la educación superior: ¿qué papel juegan los nuevos regionalismos en la difusión de estas ideas? El caso del MERCOSUR (1992-2012) en perspectiva comparada", en Revista Iberoamericana de Educación Superior (RIES), México, UNAM-IISUE/Universia, vol. VI, núm. 16, pp. 90-109, https://ries.universia.net/article/view/1257/transnacionalizacion-educacion-superior-que-papel-juegan-regionalismos-difusion-ideas-caso-mercosur-1992-1912-perspectiva-comparada [consulta: fecha de última consulta]. 